\title{
Statistical equilibrium in simple exchange games I
}

\author{
E. Scalas ${ }^{1, a}$, U. Garibaldi ${ }^{2, b}$, and S. Donadio ${ }^{3, c}$ \\ 1 Laboratory on Complex Systems. Dipartimento di Scienze e Tecnologie Avanzate, Università del Piemonte Orientale "Amedeo \\ Avogadro", Via Bellini 25 G, 15100 Alessandria, Italy \\ 2 IMEM-CNR, Dipartimento di Fisica, Università di Genova, Via Dodecaneso 33, 16146 Genova, Italy \\ 3 INFN, Dipartimento di Fisica, Università di Genova, Via Dodecaneso 33, 16146 Genova, Italy
}

Eur. Phys. J. B 53, 267 (2006)

Received 8 November 2007

Published online 8 December 2007 - (C) EDP Sciences, Società Italiana di Fisica, Springer-Verlag 2007

\begin{abstract}
This erratum corrects a mistake in reference [E. Scalas, U. Garibaldi, S. Donadio, Eur. Phys. J. B 53, 267 (2006)]. In that paper, we needed an aperiodic version of the BDY game, but, in formula (1), we incorrectly presented a periodic transition matrix of period 2 in the special case of $g=2$ agents. Here, we present the right aperiodic version.
\end{abstract}

PACS. 02.50.-r Probability theory, stochastic processes, and statistics - 02.50.Ey Stochastic processes 05.40.-a Fluctuation phenomena, random processes, noise, and Brownian motion 05.40.JcBrownian motion

In Section 3.1 of reference [1], we described a stochastic game that we named the Bennati-Dragulescu-Yakovenko (BDY) game. We described two versions of the game differing by a term $1 / g$ vs. $1 /(g-1)$ in the transition probability, but we wrote the following transition matrix:

$$
P\left(\mathbf{n}_{i}^{j} \mid \mathbf{n}\right)=\frac{1-\delta_{n_{i}, 0}}{g-z_{0}(\mathbf{n})} \frac{1-\delta_{i j}}{g-1}
$$

where $\mathbf{n}$ is the occupation vector $\left(n_{1}, \ldots, n_{g}\right), n_{i}$ is the number of objects in category $i$ and $z_{0}(\mathbf{n})$ is the number of categories without objects. The meaning of this equation is simply that, in the BDY game, the loser is selected by chance among all the agents having at least one coin and the winner is randomly selected among all the other agents. For $g=2$ categories, the chain is periodic of period $d=2$, while for $g>2$ the chain is aperiodic. An aperiodic version of the game for any $g$ has the following transition

\footnotetext{
a e-mail: enrico.scalas@mfn.unipmn.it

URL: www.mfn.unipmn.it/ ${ }^{\text {scalas }}$

b e-mail: garibaldi@fisica.unige.it

c e-mail: donadio@fisica.unige.it
}

matrix:

$$
P\left(\mathbf{n}_{i}^{j} \mid \mathbf{n}\right)=\frac{1-\delta_{n_{i}, 0}}{g-z_{0}(\mathbf{n})} \frac{1}{g},
$$

meaning that the winner may also coincide with the loser. In this case there is a non-vanishing probability that the next state of the chain coincides with the previous one. Notice that the stationary distributions of the periodic and aperiodic versions do coincide. Let us now consider the case $g=n=3$. The total number of occupation states is 10 :

$$
\begin{array}{r}
(0,0,3) ;(0,3,0) ;(3,0,0) ;(1,1,1) ;(0,1,2) ;(1,0,2) \\
(1,2,0) ;(0,2,1) ;(2,0,1) ;(2,1,0) .
\end{array}
$$

The transition matrix between these states can be directly computed by using the rules of the game. For instance, the state $(0,0,3)$ can only go into the three states $(0,1,2),(1,0,2)$ and $(0,0,3)$ with equal probability $1 / 3$. The state $(0,1,2)$ can go into the five states $(1,0,2),(0,2,1),(1,1,1),(0,0,3)$, and $(0,1,2)$ and each final state can be reached with probability $1 / 6$ except for state $(0,1,2)$ which can be reached twice over six times and has probability $1 / 3$. These considerations lead to the 
following $10 \times 10$ transition matrix for the always aperiodic case:

$$
\begin{gathered}
\mathbf{P}= \\
\left(\begin{array}{cccccccccc}
1 / 3 & 0 & 0 & 0 & 1 / 3 & 1 / 3 & 0 & 0 & 0 & 0 \\
0 & 1 / 3 & 0 & 0 & 0 & 0 & 1 / 3 & 1 / 3 & 0 & 0 \\
0 & 0 & 1 / 3 & 0 & 0 & 0 & 0 & 0 & 1 / 3 & 1 / 3 \\
0 & 0 & 0 & 1 / 3 & 1 / 9 & 1 / 9 & 1 / 9 & 1 / 9 & 1 / 9 & 1 / 9 \\
1 / 6 & 0 & 0 & 1 / 6 & 1 / 3 & 1 / 6 & 0 & 1 / 6 & 0 & 0 \\
1 / 6 & 0 & 0 & 1 / 6 & 1 / 6 & 1 / 3 & 0 & 0 & 1 / 6 & 0 \\
0 & 1 / 6 & 0 & 1 / 6 & 0 & 0 & 1 / 3 & 1 / 6 & 0 & 1 / 6 \\
0 & 1 / 6 & 0 & 1 / 6 & 1 / 6 & 0 & 1 / 6 & 1 / 3 & 0 & 0 \\
0 & 0 & 1 / 6 & 1 / 6 & 0 & 1 / 6 & 0 & 0 & 1 / 3 & 1 / 6 \\
0 & 0 & 1 / 6 & 1 / 6 & 0 & 0 & 1 / 6 & 0 & 1 / 6 & 1 / 3
\end{array}\right) .
\end{gathered}
$$

Either by inspection of the matrix or by building a graph connecting the states, it can be seen that any state leads to any other state, so that this chain is irreducible. Moreover, the period of the chain is one, as $P(x, x) \neq 0$ for any state $x$. Therefore this is a finite, irreducible and aperiodic
Markov chain. There is a unique stationary distribution which is also an equilibrium distribution.

What we wrote in Section 3.1 is consistent with the always aperiodic version of the chain and not with the possibly (for $g=2$ ) periodic case. In particular, the discussion between equations (1) and (2) is only valid in the aperiodic case. In the periodic version, the stationary distribution presented in equations (2) and (3) is not the equilibrium distribution - a point which is essential for the rest of the paper. Hence, in equation (1) and in the discussion before equation (2), $g-1$ must be replaced by $g$. These remarks correct all the mistakes in reference [1].

One of the authors (E.S.) wishes to thank F. Rapallo for fruitful discussion.

\section{References}

1. E. Scalas, U. Garibaldi, S. Donadio, Eur. Phys. J. B 53, 267 (2006) 